\title{
A CONVERSE TO GAUSS' THEOREM
}

\author{
BY WILLIAM A. VEECH ${ }^{1}$
}

Communicated by Harry Kesten, November 11, 1971

The purpose of this note is to outline a proof of the following converse to the mean value theorem for harmonic functions in two variables. Details will appear in [9].

THEOREM 1. Let $\Omega$ be a bounded Lipschitz domain in the plane, and let $f$ be a Lebesgue measurable function on $\Omega$ such that $|f(x)| \leqq g(x), x \in \Omega$, for some positive harmonic function $g$ on $\Omega$. If for each $x \in \Omega$ there is a disc contained in $\Omega$ and centered at $x$ over which the average of $f$ is $f(x)$, and if $\delta(x)$, the radius of this disc, as a function of $x$ is bounded away from 0 on compact subsets of $\Omega$, then $f$ is harmonic.

Our study has been motivated by the special case of Theorem 1 stated by Feller [5] in which $\Omega$ is the unit disc, $f$ is bounded, and $\delta(x)=d\left(x, \Omega^{c}\right)$ is the distance from $x$ to the complement of $\Omega$. A proof of Feller's assertion appears in [1]. In [2], Baxter obtains the conclusion of harmonicity in any dimension under the following assumptions: (a) $\Omega$ is a $C^{1}$ manifold with boundary, (b) $\delta(\cdot)$ is measurable, (c) $f$ is bounded, and (d) for some constant $c>0, \delta(x) \geqq c d\left(x, \Omega^{c}\right)$. In a later paper [6], Heath obtains the same conclusion under assumptions (b), (c), (a') $\Omega$ is a bounded region in $\boldsymbol{R}^{m}$, and $\left(\mathrm{d}^{\prime}\right)$ for some constant $c>0,(1-c) d\left(x, \Omega^{c}\right) \geqq \delta(x) \geqq c d\left(x, \Omega^{c}\right)$.

Our approach to Theorem 1, outlined below, differs from the papers cited above, although [6] also uses a probabilistic argument. The extension of Theorem 1 to $n \geqq 3$ dimensions is almost immediate once our "density theorem," Theorem 3 , has been so extended.

Below $\psi(h, \delta, x), x \in \Omega, 0<\delta \leqq d\left(x, \Omega^{c}\right)$, denotes the average of the measurable function $h$ over the disc, $B_{\delta}(x)$, of radius $\delta$ centered at $x$. The assumption in Theorem 1 becomes $\psi(f, \delta(x), x)=f(x), x \in \Omega$.

LEMMA 1. If $f, \delta$ and $g$ are as in Theorem 1, there exist Borel functions $f_{0}$ and $\delta_{0}$ such that $f_{0}=f$ a.e. on $\Omega, \delta_{0}(x) \geqq \delta(x), x \in \Omega,\left|f_{0}\right| \leqq g$, and $\psi\left(f_{0}, \delta_{0}(x), x\right)=f_{0}(x), x \in \Omega$.

If $f_{0}$ can be proved harmonic, then because $f=f_{0}$ almost everywhere, $f(x)=\psi(f, \delta(x), x)=\psi\left(f_{0}, \delta(x), x\right)=f_{0}(x), x \in \Omega$, and $f$ is harmonic. We are thus free to assume both $f$ and $\delta$ Borel.

AMS 1970 subject classifications. Primary 31C99; Secondary $60 \mathrm{~J} 45$.

Key words and phrases. Harmonic, Lipschitz domain, invariant $\sigma$-field, martingale.

${ }^{1}$ Sloan foundation fellow. Research in part supported by NSF-GP-18961. 
Define a Markov transition operator $P(x, y)$ by $P(x, y)=m\left(B_{\delta}(x)\right)^{-1}$, $y \in B_{\delta}(x), \delta=\delta(x)$, and $P(x, y)=0, y \notin B_{\delta}(x)$. Here $m(\cdot)$ denotes Lebesgue measure. Following Feller we use the positive harmonic function $g$ in Theorem 1 to construct a second transition operator

$$
P_{g}(x, y)=g(x)^{-1} P(x, y) g(y)
$$

Let $\mathscr{X}=\Omega \times \Omega \times \ldots$ with coordinate functions $x_{n}, n=1,2, \ldots$, and product Borel $\sigma$-field $\mathscr{B}=\mathscr{B}\left(x_{1}, x_{2}, \ldots\right)$. On $\mathscr{B}$ we define a probability measure $\mu_{x}^{g}$ which realizes the Markov chain starting at $x \in \Omega$ and governed by $P_{\mathrm{g}}$. Let $\mathscr{B}_{I} \subseteq \mathscr{B}$ be the sets representable as $E=\Omega \times E$, or what is the same, $E=\sigma E=\sigma^{-1} E$, where $\sigma$ is the left shift on $\chi . \mathscr{B}_{I}$, the "invariant $\sigma$-field," is a sub- $\sigma$-field of $\mathscr{B}_{\infty}=\bigcap_{n} \mathscr{B}\left(x_{n}, x_{n+1}, \ldots\right)$, the "tail- $\sigma$-field." The following lemma is very easy.

LEMMA 2. For any pair $x, y \in \Omega$ the measures $\mu_{x}^{g}, \mu_{y}^{g}$ are mutually absolutely continuous on $\mathscr{B}_{I}$.

REMARK. With additional assumptions on $\delta$, e.g. (d) above or $\delta$ uniformly Lipschitz on $\Omega$, we can prove mutual absolute continuity on $\mathscr{B}_{\infty}$. We cannot thus far prove mutual absolute continuity on $\mathscr{B}_{\infty}$ for arbitrary $\delta$.

The function $F(x)=f(x) / g(x), x \in \Omega$, is a bounded Borel solution to $P_{g} F=F$, and therefore the process $F_{n}(\omega)=F\left(x_{n}(\omega)\right)$ is a bounded $\mu_{x}^{g}$ martingale for any starting point $x$. By the martingale theorem and Lemma 2 there exists a $\mathscr{B}_{I}$ measurable function $F_{\infty}$ such that $\lim _{n} F_{n}(\omega)=F_{\infty}(\omega)$, a.e. $\mu_{x}^{g}$, and $F(x)=\int_{x} F_{\infty}(\omega) \mu_{x}^{8}(d \omega)$, all $x \in \Omega$. Noting that $F_{\infty}$ is uniformly approximable by linear combinations of characteristic functions of $\mathscr{B}_{1}$ sets, and that $g(x) F(x)=f(x)$, we have that $f(x)$ is locally uniformly approximable by linear combinations of functions of the form $g(x) \mu_{x}^{8}(E), E \in \mathscr{B}_{I}$. Therefore,

LEMMA 3. If $g(x) \mu_{x}^{8}(E)$ is harmonic for all $E \in \mathscr{B}_{I}$, then $f$ is harmonic.

The relationship between $\mathscr{B}_{I}$ and solutions to $P f=f$ was first studied in the case of countable state Markov chains by Blackwell [3].

Fix any point $x_{0} \in \Omega$ and let $\mathscr{M}(\Omega)$ be the extreme points of the set of positive harmonic functions on $\Omega$ which assume the value 1 at $x_{0}$. There exists a finite Borel measure $\Lambda$ on $\mathscr{M}$ such that $g(x)=\int_{\mathscr{M}} h(x) \Lambda(d h), x \in \Omega$ [8]. From this representation it follows by a simple argument that

$$
g(x) \mu_{x}^{g}(E)=\int_{\mathscr{M}} h(x) \mu_{x}^{h}(E) \Lambda(d h)
$$

holds for all $E \in \mathscr{B}$. If $E \in \mathscr{B}_{1}$, and if $h$ and $x$ are such that $\mu_{x}^{h}(E)=0$ (resp. 1), then for all $y \in \Omega, \mu_{y}^{h}(E)=0$ (resp. 1) by Lemma 2. If there exists some $x$ 
such that for all $h \in \mathscr{M}, \mu_{x}^{h}(E)=0$ or 1 , then the Borel set $E_{0}=\left\{h \mid \mu_{x}^{h}(E)\right.$ $=1\}$ will be such that for all $x$

$$
g(x) \mu_{x}^{g}(E)=\int_{E_{0}} h(x) \Lambda(d h)
$$

and $g(x) \mu_{x}^{g}(E)$ is harmonic. Theorem 1 is thus a consequence of

THEOREM 2. If $\Omega$ is a bounded Lipschitz domain in the plane, then $\mathscr{B}_{I}$ is $\mu_{x}^{g}$ trivial for every $g \in \mathscr{M}(\Omega)$ and $x \in \Omega$.

Space does not permit an outline of the proof of Theorem 2. The proof uses results from potential theory (particularly [7]) and probability theory. Another important ingredient is the following theorem which may be of independent interest.

THEOREM 3. There exists a function $\varphi(\beta)>0,0<\beta \leqq 1$, with the following property. If $S$ is the unit square in the plane, and if $A \subseteq S$ is Lebesgue measurable with $m(A)=\beta>0$, there exists a point $x \in A$ such that $m(A \cap Q)$ $\geqq \varphi(\beta) m(Q)$ for every square $Q$ such that $x \in Q \subseteq S$.

We obtain $\varphi(\beta)>2^{-288} \beta^{36}$, but the exponent 36 can be lowered, at least below 25. If $Q$ is required to have sides parallel to the axes, the exponent drops to 6, and simple examples show it can be no lower than 2 .

\section{REFERENCES}

1. M. A. Akcoglu and R. W. Sharpe, Ergodic theory and boundaries, Trans. Amer. Math. Soc. 132 (1968), 447-460. MR 37 \#369.

2. J. R. Baxter, Restricted mean values and hurmonic functions, University of Minnesota, Minneapolis, Minn. (preprint).

3. D. H. Blackwell, On transient Markou processes with a countable number of states and stationary transition probabilities, Ann. Math. Statist. 26 (1955), 654-658. MR 17, 754.

4. J. L. Doob, Stochastic processes, Chapman \& Hall, London; Wiley, New York, 1953. MR 15, 445.

5. W. K. Feller, Boundaries induced by non-negative matrices, Trans. Amer. Math. Soc. 83 (1956), 19-54. MR 19, 892.

6. D. Heath, Functions possessing restricted mean value properties, University of Minnesota, Minneapolis, Minn. (preprint).

7. R. Hunt and R. Wheeden, Positive harmonic functions on Lipschitz domains, Trans. Amer. Math. Soc. 147 (1970), 507-527.

8. R. S. Martin, Minimal positive harmonic functions, Trans. Amer. Math. Soc. 49 (1941), 137-172. MR 2, 292.

9. W. A. Veech, The core of a measurable set and a problem in potential theory (submitted).

Department of Mathematics, Rice University, Houston, Texas 77001 